\title{
A time-optimal aircraft-following model based on Pontryagin's minimum principle
}

\author{
Linghang $\mathrm{MENG}^{1 *}$, Xiaohao $\mathrm{XU}^{2}$, Zengxian $\mathrm{GENG}^{1}$ \\ 1. College of Air Traffic Management, Civil Aviation University of China, Tianjin 300300, China \\ 2. Air Traffic Management Research Base, Civil Aviation University of China, Tianjin 300300, China
}

\begin{abstract}
A time-optimal aircraft-following model is introduced to address air traffic flow interference by velocity reduction. The objective function is set up as minimizing the recovery time during which the separation minima are not infringed and the separation of the air traffic flow returns to the initial separation at the terminal time. Pontryagin's minimum principle is used to solve the optimum aircraft-following velocity control law. An analytical minimum safe following separation is also provided under the time-optimal control law. The simulation results show that the precision first-order tracking accuracy is achieved without losing the separation.
\end{abstract}

Key words: air traffic flow; aircraft-following model; time-optimal control; safe following separation

(C) 2011 JMT. All rights reserved.

\section{Introduction}

$\mathrm{T}$ he increased air traffic flow forces aircraft to operate under a far more compacted formation. Air traffic controllers' workload will in turn increase greatly in current centralized air traffic control (ATC) environment. Fortunately, information exchange on aircraft positions, velocities, and pilots' intentions among airborne agents has been promoted by using the techniques of automatic dependent surveillance-broadcast (ADS-B) [1], cockpit display of traffic information (CDTI), and traffic information services broadcast (TIS-B) [2]. These techniques enable optimal allocation of responsibilities, workload, and risk among all agents of air traffic systems. This allocation scheme proposes a decentralization of ATC system under which pilots are accorded more rights in determining their own coordinated trajectory [3-4]. As a result, a new operating concept, airborne separation assurance system (ASAS) [5-6] was introduced as a key feature in future decentralized ATC environments. ASAS is an integrated air-to-air and air-toground system which enables flight crew to maintain airborne separation by visualizing surrounding air traffic information in a cockpit display. It also allows crew to handle traditional tasks of air traffic controllers. Multi-

Received Sep. 22, 2011; revision accepted Dec. 7, 2011

${ }^{*}$ Corresponding author. E-mail: lhmeng@cauc.edu.cn (L.H. MENG)

(C) 2011 JMT. All rights reserved

doi: 10.3969/j.issn.2095-087X.2011.04.008 ple aircraft following flights will eventually form a lineshaped pattern en route or in terminal area. Under this background, some scholars tried to study air traffic management in light of air traffic flow, such as flow corridors [7], skyways [8], and tubes [9-11]. These studies that elaborated on dynamic airspace configuration, also provided insights into a tube-based operation concept. Menon [12] advanced an Eulerian approach to model air traffic flow. Strub [13], Sridhar [14], and Saraf [15] used improved Eulerian methods to solve air traffic flow management problems. This modeling technique spatially aggregated air traffic to generate models of air traffic flow on a macro aspect; however, formation of line-shaped patterns by pilots via aircraft velocity adjustment was not discussed.

In this paper, we focus on aircraft's following performance which is crucial to the safety of line-shaped pattern operation in future decentralized ATC environment. Characteristic requirements of line-shaped pattern of air traffic are first analyzed. Subsequently a timeoptimal control model with the interference of velocity reduction was developed. Pontryagin's minimum principle was then used to solve the optimal velocity control law for the aircraft following. An analytical minimum safe following separation was also derived from the time-optimal control law. Finally, the real performance data were used to simulate the model.

\section{Line-shaped pattern air traffic}

A robust line-shaped pattern flight can be assembled 
by the aircraft with comparative flight envelope flying on the same route legs. Each aircraft's velocity is confined to a compromising velocity of the air traffic flow. The flight separation among aircraft can recover to the initial following separation in a shortest time span, and the separation minima will not be infringed during the recovering time when the flow is interfered with the velocity reduction of the leading aircraft. The robustness here is defined as the performance of the air traffic flow in maintaining the pattern and separation during velocity adjustment of the leading aircraft.

The most accurate and desirable way to achieve this goal is to design a continuously differentiable velocity control law for the following aircraft. However, there is no feasibility for this kind of velocity control law because of the restrictions of flight manipulation. A constant deceleration control law may lead to monotonic decrease of the separation among air traffic flow. We do not expect this with our approach. We anticipate an initial rapid velocity reduction of following aircraft at a constant deceleration for the sake of following safety, followed by an increased velocity at a constant acceleration in order to achieve the initial separation. This kind of velocity control law could be achieved by controlling N1 rotation velocity of a turbojet aircraft. In this paper, we are not going to deduce the relationship between N1 rotation velocity and the deceleration of the following aircraft. We only give a theoretical equation for computing the aircraft's level flight maneuverability $a_{x}$ [16-17]:

$$
a_{x}=\frac{\mathrm{d} v}{\mathrm{~d} t}=\frac{\left(F_{N}-D\right)}{W} g=n_{x} g,
$$

where $F_{N}$ is the available thrust which relies on N1 rotation velocity, $D$ denotes the drag which relies on the velocity and configuration of the aircraft, $\left(F_{N}-D\right)$ denotes the available residual thrust of the aircraft, and $n_{x}$ denotes the overload factor of the longitudinal axis during level flight.

\section{Time-optimal aircraft-following model}

Based on the analysis in Section 2, the problem could be identified as a time-optimal following problem with a fixed start point and a free terminal point [18]. Therefore, the performance function is established as

$$
\min J[u(t)]=\int_{\tau}^{t_{\mathrm{f}}} \mathrm{d} t,
$$

where $J(\cdot)$ is the recovery time of the following aircraft under the velocity reduction interference of the leading aircraft, $u(t)$ is the deceleration of the following aircraft at time $t, \tau$ denotes the total reaction delay of the fol- lowing aircraft, and $t_{\mathrm{f}}$ is the terminal time when the following aircraft keeps in step with the leading aircraft. Let $x_{1}(t)$ and $x_{2}(t)$ be the position and velocity of the following aircraft, respectively. Then the state equations could be expressed as

$$
\begin{aligned}
& \dot{x}_{1}(t)=x_{2}(t), \\
& \dot{x}_{2}(t)=u(t) .
\end{aligned}
$$

The initialization conditions are

$$
\begin{aligned}
& x_{1}(\tau)=v_{0} \tau, \\
& x_{2}(\tau)=v_{0} .
\end{aligned}
$$

For the leading aircraft $i$ at time 0 , braking action is taken which forces the aircraft $i$ to reduce velocity from the initial velocity of the air traffic flow $v_{0}$ to the terminal velocity $v_{\infty}$ of the air traffic flow at a constant deceleration $a_{i}$ until time $t_{\infty}$, where $t_{\infty}$ could be calculated by

$$
t_{\infty}=\frac{v_{\infty}-v_{0}}{a_{i}} .
$$

Then the motion equation of the leading aircraft could be deduced as

$$
x_{i}(t)=\left\{\begin{array}{cc}
s_{0}+v_{0} t+\frac{1}{2} a_{i} t^{2}, & t<t_{\infty}, \\
s_{0}+\frac{v_{\infty}^{2}-v_{0}^{2}}{2 a_{i}}+v_{\infty}\left(t-t_{\infty}\right), & t \geq t_{\infty},
\end{array}\right.
$$

where $x_{i}(t)$ represents the position of leading aircraft $i$ at time $t$, and $s_{0}$ denotes the initial separation. The firstorder tracking accuracy shall be assured by the terminal constraints, namely the following aircraft shall keep flight separation from the leading aircraft at a distance of $s_{0}$ and maintain the same terminal velocity of $v_{\infty}$ with the leading aircraft at the terminal time $t_{\mathrm{f}}$. The terminal constraints are listed as follows:

$$
\begin{aligned}
& x_{1}\left(t_{\mathrm{f}}\right)=x_{i}\left(t_{\mathrm{f}}\right)-s_{0}, \\
& x_{2}\left(t_{\mathrm{f}}\right)=v_{\infty},
\end{aligned}
$$

where $x_{1}\left(t_{\mathrm{f}}\right)$ is the following aircraft's position at time $t_{\mathrm{f}}$, and $x_{2}\left(t_{\mathrm{f}}\right)$ is its velocity at time $t_{\mathrm{f}}$.

\section{Solution of the model}

In this section, we use Pontryagin's minimum principle [18] to solve the time-optimal control model 
proposed in this paper. Pontryagin's minimum principle is used in optimal control theory to find the best possible control to drive a dynamical system from one state to another, especially in the presence of constraints for the state or input controls. The principle states informally that the Hamilton's function must be minimized over the set of all permissible controls. In our model, the Hamilton's function is given as

$$
H=1+\lambda_{1}(t) x_{2}(t)+\lambda_{2}(t) u(t),
$$

where $\left[\begin{array}{ll}\lambda_{1}(t) & \lambda_{2}(t)\end{array}\right]^{\mathrm{T}}$ is the adjoint vector. A group of necessary conditions can be deduced using Pontryagin's minimum principle as follows:

(a) Canonical equations:

$$
\begin{aligned}
& \dot{x}_{1}(t)=\frac{\partial H}{\partial \lambda_{1}(t)}=x_{2}(t), \\
& \dot{x}_{2}(t)=\frac{\partial H}{\partial \lambda_{2}(t)}=u(t), \\
& \dot{\lambda}_{1}(t)=-\frac{\partial H}{\partial x_{1}(t)}=0, \\
& \dot{\lambda}_{2}(t)=-\frac{\partial H}{\partial x_{2}(t)}=-\lambda_{1}(t) .
\end{aligned}
$$

(b) Conditional extremum:

$$
\begin{aligned}
& H\left(x_{1}^{*}, x_{2}^{*}, u^{*}, t\right)= \\
& \quad \min \left[1+\lambda_{1}(t) x_{2}(t)+\lambda_{2}(t) u(t)\right] .
\end{aligned}
$$

(c) Constraints of control:

$$
a_{\min } \leq u(t) \leq a_{\max },
$$

where $a_{\min }$ and $a_{\text {max }}$ denote the minimum and maximum of reasonable extreme accelerations limited by the flight envelope of the following aircraft, respectively;

(d) Initial conditions:

$$
\begin{aligned}
& x_{1}(\tau)=v_{0} \tau, \\
& x_{2}(\tau)=v_{0} .
\end{aligned}
$$

(e) Terminal conditions:

$$
\begin{aligned}
& \xi_{1}\left(t_{\mathrm{f}}^{*}\right)=x_{1}\left(t_{\mathrm{f}}^{*}\right)-\frac{\left(v_{\infty}\right)^{2}-\left(v_{0}\right)^{2}}{2 a_{i}}- \\
& v_{\infty}\left(t_{\mathrm{f}}^{*}-t_{\infty}\right)=0, \\
& \xi_{2}\left(t_{\mathrm{f}}^{*}\right)=x_{2}\left(t_{\mathrm{f}}^{*}\right)-v_{\infty}=0 .
\end{aligned}
$$

The solutions in the forms of best phase locus and optimal control law could be obtained by solving Eqs. (12) to $(21)$.

The best phase locus is

$$
x_{1}^{*}(t)=\left\{\begin{array}{l}
\frac{\left(x_{2}^{*}(t)\right)^{2}}{2 a_{\min }}+v_{0} \tau-\frac{\left(v_{0}\right)^{2}}{2 a_{\min }}, \\
\tau \leq t<t_{\mathrm{a}}^{*}, \\
\frac{\left(x_{2}^{*}(t)\right)^{2}}{2 a_{\max }}+v_{\infty} t_{\mathrm{f}}^{*}-\frac{\left(v_{\infty}\right)^{2}}{2 a_{\max }}-\frac{\left(v_{\infty}-v_{0}\right)^{2}}{2 a_{i}}, \\
\quad t_{\mathrm{a}}^{*} \leq t<t_{\mathrm{f}}^{*},
\end{array}\right.
$$

where $t_{\mathrm{a}}^{*}$ is the optimal switch time, and $t_{\mathrm{f}}^{*}$ is the optimal terminal time. The optimal control law is obtained as

$$
u^{*}(t)= \begin{cases}a_{\min }, & \tau \leq t<t_{\mathrm{a}}^{*}, \\ a_{\max }, & t_{\mathrm{a}}^{*} \leq t<t_{\mathrm{f}}^{*},\end{cases}
$$

where

$$
\begin{aligned}
& t_{\mathrm{a}}^{*}=\frac{z-\sqrt{z^{2}-4(x-y)\left(a_{\max } a_{\text {min }}-\left(a_{\text {min }}\right)^{2}\right)}}{2\left[a_{\text {max }} a_{\text {min }}-\left(a_{\text {min }}\right)^{2}\right]}, \\
& t_{\mathrm{f}}^{*}=\frac{v_{\infty}+a_{\text {max }} t_{\mathrm{a}}-a_{\text {min }}\left(t_{\mathrm{a}}-\tau\right)-v_{0}}{a_{\text {max }}},
\end{aligned}
$$

and

$$
\begin{aligned}
& x=a_{\text {max }} a_{\text {min }} \tau^{2}-2 v_{0} a_{\text {min }} \tau^{2}+\frac{a_{\text {max }}\left(v_{\infty}-v_{0}\right)^{2}}{a_{i}}, \\
& y=\left(v_{\infty}\right)^{2}+\left(v_{0}\right)^{2}+\left(a_{\text {min }}\right)^{2} \tau^{2}, \\
& z=2\left(a_{\text {max }}-a_{\text {min }}\right)\left(v_{\infty}-a_{\text {min }} \tau\right) .
\end{aligned}
$$

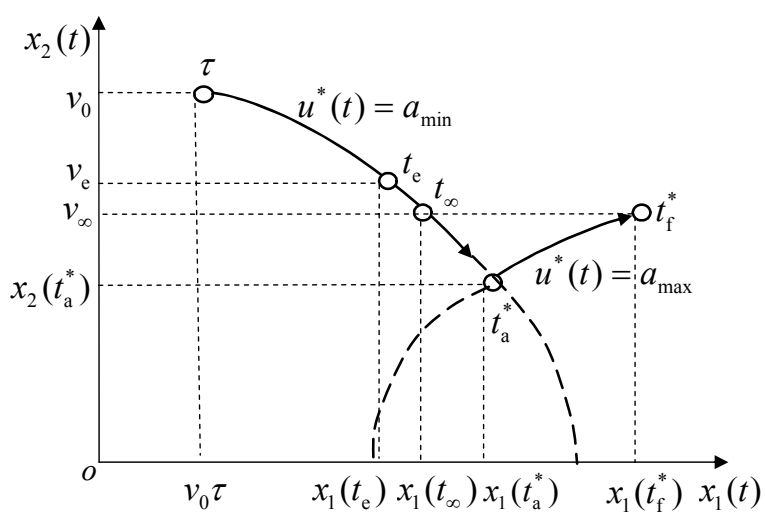

Fig. 1 The optimal phase locus

Fig. 1 shows the best phase locus derived by the time-optimal control law. Since the following aircraft reduce its velocity at a bigger deceleration than the leading aircraft, in order to make sure a certain level of safety margin, there shall be a specific time $t_{\mathrm{e}}\left(t_{\mathrm{e}} \leq t_{\infty}\right.$ and $\left.x\left(t_{\infty}\right)=v_{\infty}\right)$ at which both aircraft are at the same 
velocity of $v_{\mathrm{e}}\left(v_{\mathrm{e}} \geq v_{\infty}\right)$, which means the separation between them drops to the minimum value at $t_{\mathrm{e}}$. Therefore, the initial safe following separation $s_{0}$ should be assured by inequality $s\left(t_{e}\right) \geq s_{\text {atc }}$, where $s_{\text {atc }}$ denotes the ATC minimum separation. Then $s_{0}$ can be calculated from the following inequality:

$$
\begin{aligned}
& s_{0} \geq s_{\text {atc }}+\frac{1}{2} a_{\min }\left(\frac{a_{\text {min }} \tau}{a_{\text {min }}-a_{i}}-\tau\right)^{2}- \\
& \frac{1}{2} a_{i}\left(\frac{a_{\text {min }} \tau}{a_{\text {min }}-a_{i}}\right) \cdot{ }^{2}
\end{aligned}
$$

\section{Simulation results}

In simulation, the flight maneuverability for civil aircraft is confined between $-1.0 \mathrm{~g}$ and $2.5 \mathrm{~g}$ [17-18]. Considering that the aircraft will operate with the compromising performance under a line-shaped pattern traffic flow, and from energy savings consideration, the aircraft will not adopt the boundary deceleration or acceleration. Thus, we employ a reasonable level flight maneuverability as $a_{\min }=-0.5 g$ and $a_{\max }=g$. And other simulation parameters are listed in Table 1 . The simulation results calculated are shown in Table 1 and Figs. 2 to 4.

\begin{tabular}{|c|c|c|c|c|c|c|c|c|c|}
\hline $\begin{array}{c}S_{\text {atc }} \\
(\mathrm{km})\end{array}$ & $\begin{array}{c}v_{0} \\
(\mathrm{~km} / \mathrm{h})\end{array}$ & $\begin{array}{c}a_{\max } \\
(g)\end{array}$ & $\begin{array}{l}a_{\text {min }} \\
(g)\end{array}$ & $\begin{array}{c}a_{i} \\
(g)\end{array}$ & $\begin{array}{c}v_{\infty} \\
(\mathrm{km} / \mathrm{h})\end{array}$ & $\tau(\mathrm{s})$ & $t_{\mathrm{a}}^{*}(\mathrm{~s})$ & $t_{\mathrm{f}}^{*}(\mathrm{~s})$ & $s_{0}(\mathrm{~km})$ \\
\hline \multirow{32}{*}{10} & \multirow{32}{*}{550} & \multirow{32}{*}{1} & \multirow{32}{*}{-0.5} & \multirow{16}{*}{-0.25} & \multirow{4}{*}{500} & 2 & 6.3185 & 7.0606 & 10.010 \\
\hline & & & & & & 3 & 8.2802 & 9.5031 & 10.022 \\
\hline & & & & & & 4 & 9.9587 & 11.5209 & 10.039 \\
\hline & & & & & & 5 & 11.5142 & 13.3540 & 10.061 \\
\hline & & & & & \multirow{4}{*}{510} & 2 & 5.8859 & 6.6950 & 10.010 \\
\hline & & & & & & 3 & 8.8306 & 7.6429 & 10.022 \\
\hline & & & & & & 4 & 9.2113 & 10.6832 & 10.039 \\
\hline & & & & & & 5 & 10.6865 & 12.3960 & 10.061 \\
\hline & & & & & \multirow{4}{*}{520} & 2 & 5.3153 & 6.1226 & 10.010 \\
\hline & & & & & & 3 & 6.9085 & 8.0124 & 10.022 \\
\hline & & & & & & 4 & 8.3731 & 9.7094 & 10.039 \\
\hline & & & & & & 5 & 9.7682 & 11.3020 & 10.061 \\
\hline & & & & & \multirow{4}{*}{530} & 2 & 4.6057 & 5.3416 & 10.010 \\
\hline & & & & & & 3 & 6.0516 & 7.0106 & 10.022 \\
\hline & & & & & & 4 & 7.4119 & 8.5510 & 10.039 \\
\hline & & & & & & 5 & 8.7225 & 10.0169 & 10.061 \\
\hline & & & & \multirow{16}{*}{-0.35} & \multirow{4}{*}{500} & 2 & 7.1286 & 8.2757 & 10.023 \\
\hline & & & & & & 3 & 8.8415 & 10.3451 & 10.051 \\
\hline & & & & & & 4 & 10.4152 & 12.2056 & 10.091 \\
\hline & & & & & & 5 & 11.9089 & 13.9461 & 10.143 \\
\hline & & & & & \multirow{4}{*}{510} & 2 & 6.4071 & 7.4769 & 10.023 \\
\hline & & & & & & 3 & 8.0246 & 9.4031 & 10.051 \\
\hline & & & & & & 4 & 9.5271 & 11.1569 & 10.091 \\
\hline & & & & & & 5 & 10.9619 & 12.8091 & 10.143 \\
\hline & & & & & \multirow{4}{*}{520} & 2 & 5.6265 & 6.5894 & 10.023 \\
\hline & & & & & & 3 & 7.1453 & 8.3677 & 10.051 \\
\hline & & & & & & 4 & 8.5719 & 10.0075 & 10.091 \\
\hline & & & & & & 5 & 9.9428 & 11.5639 & 10.143 \\
\hline & & & & & \multirow{4}{*}{530} & 2 & 4.7636 & 5.5784 & 10.023 \\
\hline & & & & & & 3 & 6.1753 & 7.1961 & 10.051 \\
\hline & & & & & & 4 & 7.5170 & 8.7086 & 10.091 \\
\hline & & & & & & 5 & 8.8154 & 10.1563 & 10.143 \\
\hline
\end{tabular}

Table 1 Simulation results 


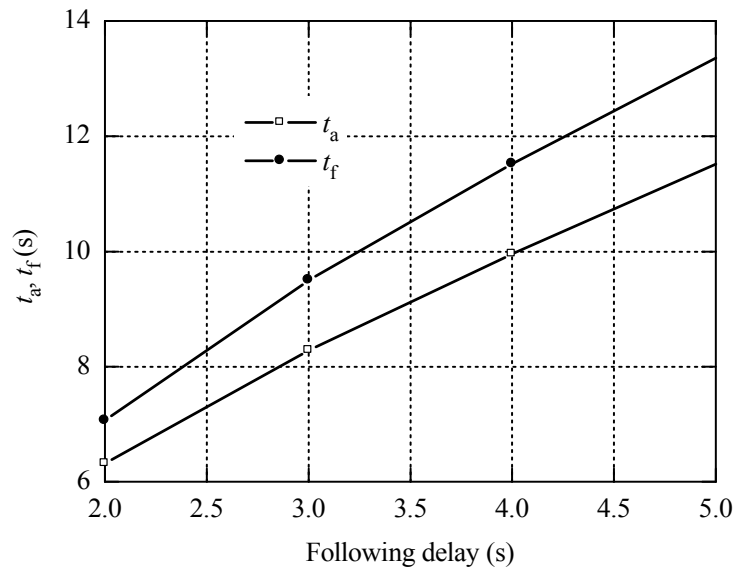

Fig. $2 t_{\mathrm{a}}^{*}$ and $t_{\mathrm{f}}^{*}$ vs. following delays, $v_{\infty}=500 \mathrm{~km} / \mathrm{h}$

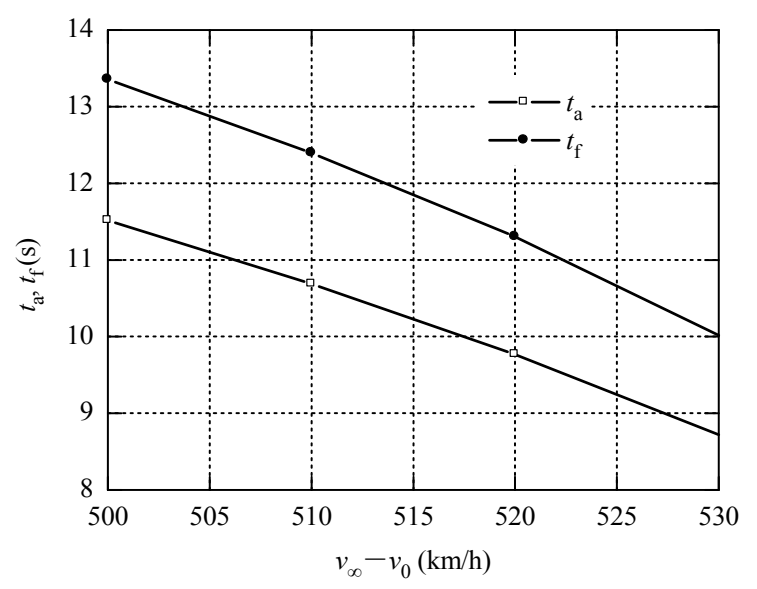

Fig. $3 t_{\mathrm{a}}^{*}$ and $t_{\mathrm{f}}^{*}$ vs. $v_{\infty}$

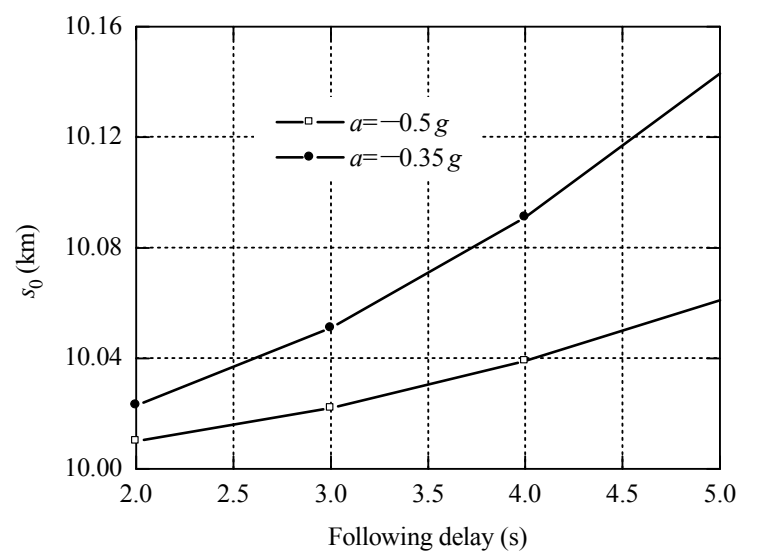

Fig. 4 Initial safe following separation vs. following delay

From the simulation results, we can draw the following conclusions:

(a) When the leading aircraft maintains a constant acceleration, the optimal switch time $t_{\mathrm{a}}^{*}$ and the optimal terminal time $t_{\mathrm{f}}^{*}$ are approximately proportional to the following delay time $\tau$ (see Fig. 2) and approxi- mately inversely proportional to the velocity reduction $\left|v_{\infty}-v_{0}\right|$ (see Fig. 3), while the initial safe following separation is nearly in positive proportion with the following delay time $\tau$ but has nothing to do with $v_{0}-v_{\infty}$ (see Fig. 4).

(b) When the leading aircraft takes a stronger braking action, there will be a lag for the optimal switch time $t_{\mathrm{a}}^{*}$ and the optimal terminal time $t_{\mathrm{f}}^{*}$.

(c) The loss of the dynamic safe following separation does not exceed the criteria. In this case, the maximum loss of the dynamic following separation is less than $200 \mathrm{~m}$. Compared with the radar separation $10 \mathrm{~km}$ en route, the dynamic safe following separation proposed by this paper could ensure no separation loss event.

\section{Conclusions and limitation}

In this paper, we propose a time-optimal control model of air traffic flow with velocity reduction interference as well as its objective function and terminal constraints. Pontryagin's minimum principle is used to design the optimal velocity control law of the following aircraft. An analytical minimum safe following separation is also given under the time-optimal control law. The simulation results show that the first-order tracking performance under velocity reduction disturbance can be achieved by using our time-optimal control law and the loss of the dynamic safe following separation does not exceed the specified criteria. Therefore, the model's validity to maintain line-shaped pattern air traffic can be guaranteed. However, it should be noted that the proposed model is not an economical one and it only makes sense to compacted air traffic flow and its optimal objective focuses on safety and fast response only.

\section{Acknowledgements}

This paper is supported by the National Natural Science Foundations of China (Nos. 60972006 and 61179042) and the National Science and Technology Support Program (No. 2011BAH24B10).

\section{References}

[1] RTCA/DO-242A, Minimum aviation system performance standards for automatic dependent surveillance broadcast (ADS-B).

[2] W. Knecht, P. Hancock, Separation maintenance in high-stress free flight using a time-to-contact-based cockpit display of traffic information, In: Proceedings of the Human Factors and Ergonomics Society of the 
43rd Annual Meeting, Houston, Texas, Sep. 27-Oct. 1, 1999: 16-20.

[3] S.M. Green, K.D. Bilimoria, M.G. Ballin, Distributed air/ground traffic management for en route flight operations, Air Traffic Control Quarterly, 2001(4): 259-285.

[4] J.C. Hill, J.K. Archibald, W.C. Stirling, et al., A multiagent system architecture for distributed air traffic control, In: Proceedings of AIAA Guidance, Navigation, and Control Conference, Washington, 2005: 1936-1946.

[5] B. BoMemaison, F. Casaux, T. Miquel, Operational assessment of co-operative ASAS applications, In: Proceedings of US/Europe TMR\&D Seminar, Orlando, 1998: 47-58.

[6] F. Casaux, B. Hasquenoph, Operational use of ASAS, In: Proceedings of US/Europe ATM R\&D Seminar, Saclay, France, Jun. 17-20, 1997: 221-226.

[7] A.D. Mundra, E.M. Simons, Self-separation Corridors, In: Proceedings of Digital Avionics Systems Conference, Dallas, Oct. 21-25, 2007: 3.C.3-1-3.C.3-11.

[8] J. Alipio, P. Castro, H. Kaing, et al., Dynamic airspace super sectors (DASS) as high-density highways in the sky for a new air traffic, In: Proceedings of the 2003 Systems and Informational Engineering Design Symposium, Virginia, 2003: 57-65.

[9] M Harris, A. Raza, Z. Rojas, et al., Preliminary design analysis of dynamic airspace super sectors (DASS), In: Proceedings of the 2006 Systems and Information Engineering Design Symposium, Charlottesville, 2006: 207-211.

[10] A. Yousefi, G.L. Donohue, L. Sherry, High-volume tube-shape sectors (HTS): a network of high capacity ribbons connecting congested city pairs, In: Proceed- ings of the 23rd Digital Avionics Systems Conference, Salt Lake City, 2004: 3.C.1-1-3.C.1-7.

[11] R. Hoffman, J. Prete, Principles of airspace tube design for dynamic airspace configuration, In: Proceedings of the 8th AIAA Aviation Technology, Integration and Operations, Anchorage, 2008: 1-17.

[12] P.K. Menon, G.D. Sweriduk, K. Bilimoria, New approach for modeling, analysis and control of air traffic flow, AIAA Journal of Guidance, Control and Dynamics, 2004, 27(5): 737-744.

[13] I.S. Strub, A.M. Bayen, Optimal control of air traffic networks using continuous flow models, In: Proceedings of AIAA Guidance, Navigation, and Control Conference and Exhibit, Keystone, Colorado, Aug. 21-24, 2006: 1700-1710.

[14] B. Sridhar, T. Soni, K. Sheth, et al., Aggregate flow model for air-traffic management, AIAA Journal of Guidance, Control and Dynamics, 2006, 29(4): 992997.

[15] A. Saraf, G. Slater, Combined Eulerian-Lagrangian two-level control system for optimal air traffic flow management, In: Proceedings of AIAA Guidance, Navigation, and Control Conference and Exhibit, Keystone, Aug. 21-24, 2006: 1711-1723.

[16] Z.H. Chen, R.P. Gu, J.J. Liu, Aircraft Performance, Beijing: Publishing House of Ordnance Industry, 2006 (in Chinese).

[17] CCAR-25-R3, General administration of civil aviation of China, 2001 (in Chinese).

[18] L.S. Pontryagin, The Mathematical Theory of Optimal Processes, New York: Gordon and Breach Science Publishers, 1987.

(Editor: Dongju CHEN) 